\title{
Conscious and unconscious influences of memory for object location
}

\author{
JUDY I. CALDWELL and MICHAEL E. J. MASSON \\ University of Victoria, Victoria, British Columbia, Canada
}

\begin{abstract}
The process-dissociation procedure was used to investigate conscious and unconscious influences of memory for object location. In two experiments, subjects worked with drawings of household objects and rooms of a house depicted on a computer monitor to simulate placing objects in various locations. Memory for object locations was tested by having subjects search for those objects. A double dissociation was obtained between estimates of conscious and unconscious influences of memory computed from equations that assumed independence between these two influences: Age-related differences were found in the estimate of conscious influences, but not in the estimate of unconscious influences, whereas manipulation of habit strength affected the unconscious estimate, but not the conscious estimate. These results were closely fit by a multinomial model assuming independence between conscious and unconscious influences of memory.
\end{abstract}

When we search for an object that we earlier placed in a particular location, our efforts are influenced by some form or forms of memory that include representations of the various episodes involving our interactions with that object. One may often have the sense that this kind of search behavior is guided by a clear awareness of the most recent episode involving the object, enabling one to go directly to the appropriate location. But on other occasions, one might search in the wrong location, perhaps because that is the usual location for the object. Or one might accidentally find the object in an unexpected location. We propose that in each of these cases, search behavior may be influenced not only by conscious recollection of an object's location (accurate or inaccurate), but also by a form of unconscious memory for its location.

The role of awareness in memory for location has been examined extensively in the context of testing the hypothesis that the location of objects is automatically encoded into memory (Hasher \& Zacks, 1979). On this account, humans are genetically prepared to process automatically certain kinds of information, including location, time, and frequency information. Hasher and Zacks argued that because such attributes are processed automatically, those attributes should be remembered even following incidental learning. Moreover, memory for those attributes should

The research reported here is based on a dissertation submitted by J.I.C. to the University of Victoria in partial fulfillment of the requirements for a $\mathrm{PhD}$ degree. Support for this work was provided by a graduate scholarship to J.I.C. from the Natural Sciences and Engineering Research Council of Canada (NSERC) and by Research Grant 7910 to M.E.J.M. from NSERC. We thank Stephen Lindsay for very helpful suggestions at various stages of this research, Roger Dixon for providing access to the pool of community-dwelling older adults who took part in Experiment 1, and Stewart Arneil for computer programming assistance. Correspondence regarding this article should be sent to M. E. J. Masson, Department of Psychology, University of Victoria, P. O. Box 3050, Victoria, BC V8W 3P5, Canada (e-mail: mmasson@ uvic.ca). be unaffected by instructions and practice, developmental trends, and conditions that decrease attentional capacity. In support of this position, a number of studies have shown that the location of objects can be remembered when encoded without intention to learn and that instructions to learn location failed to affect later memory for location (e.g., Mandler, Seegmiller, \& Day, 1977; Shadoin \& Ellis, 1992).

The strong form of the Hasher and Zacks (1979) proposal, however, is not consistent with other evidence. Under certain circumstances, intention to learn location can improve performance (Light \& Zelinski, 1983; NavehBenjamin, 1987). Moreover, memory for location can be improved by both practice and encoding strategy (NavehBenjamin, 1987, 1988; Park \& Mason, 1982). Further evidence against the Hasher and Zacks model is the finding that elderly subjects do not perform as well as younger subjects on spatial memory tasks (e.g., Light \& Zelinski, 1983; Naveh-Benjamin, 1987, 1988).

Although these results challenge the strong form of Hasher and Zacks's (1979) model of automatic encoding, they do not entirely rule out the possibility that some aspects of location information are encoded automatically. Numerous studies have demonstrated that such information is remembered at greater than chance levels under incidental learning instructions (e.g., Light \& Zelinski, 1983; Naveh-Benjamin, 1988). A plausible explanation for this pattern of results is that some aspects of location information are encoded automatically but, like other kinds of information, memory for location improves if elaborative encoding processes are employed. This account is consistent with the finding of superior memory performance when intentionalencoding instructions are used and when subjects are encouraged to use a specific encoding strategy.

The suggestion that memory for object location may involve both automatic and strategic processes fits the distinction between automatic and controlled (or uncon- 
scious and conscious) influences of memory as developed by Jacoby and his colleagues (e.g., Jacoby, 1991; Jacoby, Lindsay, \& Toth, 1992; Jennings \& Jacoby, 1993). In that formulation, conscious and unconscious influences of memory are assumed to have independent effects on task performance. On the basis of this premise, Jacoby (1991) developed the process-dissociation procedure to assess separately these two kinds of memory within a single task. This procedure separates conscious and unconscious influences by using two instructional conditions: an inclusion condition, in which conscious and unconscious influences each produce the target response, and an exclusion condition, in which the unconscious influences of memory produce the target response, but conscious influences produce an opposing response. By assuming that conscious and unconscious influences make independent contributions in determining a response, it is possible, as will be illustrated below, to generate separate estimates of these two influences.

To illustrate this, let $C$ and $U$ represent the probability that conscious and unconscious influences of memory, respectively, lead to the target response. In the inclusion condition, the probability that the target response is produced is $I=C+(1-C) U$. In the exclusion condition, the probability of generating the target response is $\mathrm{E}=(1-$ $C) U$. Both $I$ and $E$ are observed probabilities. Simple algebra allows one to solve for $C$ and $U$, which provide estimates of conscious and unconscious influences of memory. The critical assumptions underlying this procedure are the following: (1) $C$ and $U$ are independent, (2) $C$ and $U$ operate in a consistent manner in both the inclusion and the exclusion conditions, and ( 3 ) the response generated by $C$ dominates the response generated by $U$.

It is important to note that the assumptions underlying these equations, particularly the assumption that conscious and unconscious influences of memory make independent contributions to task performance, have been challenged (e.g., Curran \& Hintzman, 1995, 1997; Graf \& Komatsu, 1994; Joordens \& Merikle, 1993). For instance, Curran and Hintzman (1995) found that in a wordstem completion task, estimates of the conscious influence $(C)$ of prior study of a completion on its use when later completing a stem increased with study time, whereas estimates of unconscious influences of prior study $(U)$ decreased with study time. The results involving the estimate of $U$ were paradoxical, given previous findings that indirect tests of memory are not affected by changes in study time (Greene, 1986; Jacoby \& Dallas, 1981), implying that estimates of $U$ should likewise be unaffected by study time. Curran and Hintzman proposed that the effect of prior study on $U$ was due to violation of the independence assumption.

Jacoby, Yonelinas, and Jennings (1997) argued that although there may be circumstances under which the independence relation between $C$ and $U$ may not hold, there are many demonstrations of predicted dissociations between estimates of these influences, in keeping with the independence assumption. In most of these demonstra- tions, factors typically associated with cognitive control, such as full versus divided attention at study, elaborative encoding, and age of subjects, affected $C$ but not $U$. These outcomes are consistent with experiments that compared direct and indirect tests of memory, in which the former type of test, but not the latter, showed effects of such manipulations (e.g., Jacoby \& Dallas, 1981; Parkin, Reid, \& Russo, 1990).

Moreover, Jacoby (1998) provided evidence for the existence of boundary conditions that appear to determine whether or not $C$ and $U$ will operate independently. In particular, he proposed that direct retrieval instructions (e.g., use a word stem as a cue to recall a studied word that fits the stem), given in both inclusion and exclusion conditions, are crucial for ensuring that $C$ and $U$ operate independently and that those influences are the same in both instructional conditions (Assumptions 1 and 2, above). To test this claim, Jacoby (1998) provided some subjects with direct retrieval instructions. Other subjects were given generate-recognize instructions: In the inclusion condition, subjects were told to complete word stems with the first word that came to mind; in the exclusion condition, subjects were instructed to check each completion to be sure it was not an old word before giving it as a response. Jacoby (1998) found the expected dissociation between estimates of $C$ and $U$ when direct retrieval instructions were used, whereby dividing attention or reduced study time lowered the estimates of $C$ but did not affect the estimates of $U$. But for generate-recognize instructions, these manipulations produced the same paradoxical dissociation between $C$ and $U$ as that found by Curran and Hintzman (1995).

In addition to demonstrating different patterns of estimates of $C$ and $U$ as a function of instructions, Jacoby (1998) used two different versions of a multinomial model to fit the observed data (cf. Buchner, Erdfelder, \& Vaterrodt-Plünnecke, 1995). The direct retrieval version of the model embodied the assumption that $C$ and $U$ operated independently, and the generate-recognize version of the model was based on the assumption that subjects used a generate-recognize strategy. Each version of the model adequately fit the data from subjects given the corresponding inclusion-exclusion instructions but failed to fit the data from subjects given the alternative set of instructions. This pattern of model-fitting results further supported the idea that there are identifiable boundary conditions under which the independence assumption holds.

In the experiments reported here, we applied the process-dissociation procedure to test the hypothesis that conscious and unconscious influences of memory for the location of objects can operate independently. An earlier study by Anooshian and Seibert (1996) applied this procedure to demonstrate the independent operation of conscious and unconscious influences of memory for places that would be encountered by following a particular route through a building. Their experiments showed that estimates of conscious recollection of places was affected by divided attention but estimates of familiarity were not. 
To establish evidence for independence between conscious and unconscious influences of memory for the location of objects, we sought to obtain a double dissociation between conscious and unconscious influences of memory, in which estimates of $C$ and $U$ respond differentially, and in predictable ways, to experimental manipulations (Jacoby, 1991; Jacoby et al., 1997). In addition, we fit an independence version of a multinomial model to our observed data to determine whether such a model could be rejected. In Experiment 1, we tested for agerelated differences in conscious or in unconscious influences of memory for location. In Experiment 2, strength of habit was manipulated to determine its effect on conscious and unconscious influences of memory.

\section{EXPERIMENT 1}

In Experiment 1, we tested memory for the location of objects among younger and older adults, with the expectation that age-related differences would dissociate conscious and unconscious influences of memory. More specifically, we expected that conscious recollection of object location would be lower for older than for younger adults but that both age groups would show equal effects of unconscious influences of memory. This pattern would be consistent with the finding that performance on direct tests of memory for various kinds of events, including memory for spatial location, is superior for younger adults (e.g., Light \& Zelinski, 1983; Naveh-Benjamin, 1987, 1988), whereas older adults have been found to perform at levels comparable to younger adults on indirect tests of memory, which presumably reflect automatic or unconscious influences of memory (e.g., Light \& Singh, 1987).

Although there have been some demonstrations of a small advantage for younger over older subjects on indirect tests of memory (e.g., Chiarello \& Hoyer, 1988; Hultsch, Masson, \& Small, 1991; Small, Hultsch, \& Masson, 1995), these effects may be the result of contamination of such tests by conscious recollection. Specifically, subjects may use conscious recollection on indirect tests of memory to improve their performance even though such tests do not require them to do so. Because younger subjects demonstrate recollection that is superior to that of older adults, their indirect test performance should benefit to a greater degree if conscious recollection is involved. Jacoby and his colleagues (Jacoby, Jennings, \& Hay, 1996; Jennings $\&$ Jacoby, 1993, 1997) have used the process-dissociation procedure to compare conscious and unconscious influences of memory across younger and older subjects and have shown an advantage for younger adults on estimates of conscious influences of memory, but no age-related differences on estimates of unconscious influences. In our application of the procedure to memory for the location of objects, we expected to obtain a pattern of age-related effects similar to that found by Jacoby and his colleagues.

\section{Method}

Subjects. Thirty younger and 30 older adults participated in this experiment. The younger subjects were students at the University of
Victoria, who received extra credit in an introductory psychology course. The ages of these subjects ranged from 18 to 25 years, with a median age of 19 years. The older subjects were communitydwelling adults in the Victoria area recruited through advertisements and community groups. The subjects in this group ranged from 60 to 82 years of age, with a median age of 70 years. All the subjects were administered the 100-item vocabulary subtest of the Nelson-Denny Reading Test (Brown, Bennett, \& Hanna, 1981) to assess verbal ability. Proportion of correct scores on the vocabulary test showed a significant advantage for the older subjects $(M=$ .87 , range $=.48-1.00)$ over the younger subjects $[M=.60$, range $=$ $\left..12-.92 ; F(1,58)=37.40, M S_{\mathrm{e}}=0.030\right]$.

Materials. The stimuli consisted of 84 drawings of household objects. Twelve of these objects were used for practice trials, and the remaining 72 were used for the critical trials. Examples of these objects are shown in Figure 1. The 72 critical objects were chosen so that a different set of 12 could be associated with each of six different rooms in a house (bedroom, bathroom, kitchen, den, living room, and garage). These objects were assigned to six blocks of 12 ( 2 objects from each room) for counterbalancing purposes. For each subject, two of these blocks were assigned to the studied-inclusion condition, two were assigned to the studied-exclusion condition, one block was assigned to the nonstudied-inclusion condition, and one block was assigned to the nonstudied-exclusion condition. The assignment of the blocks to conditions was counterbalanced across subjects, with each block being assigned to each condition equally often.

Six prototypical rooms were depicted by line drawings (see Figure 2 for an example). A drawing of a floor plan was also used, which showed the layout of the six rooms corresponding to those used as backgrounds (see Figure 3). Each room in the floor plan was labeled with its name.

A pilot study was conducted to determine a target location for each object within its assigned room. In the pilot study, 10 younger adults were presented with the line drawings of the objects for a room and were asked to choose the 3 most typical locations for each object within that room. The target location for each object was chosen on the basis of the following two criteria. First, the target location was nominated by more than 1 of the 10 subjects as one of their three choices. Second, the target locations were chosen so that as many different locations as possible within each room were assigned objects. The number of locations used within the rooms ranged from 8 to 14, with a maximum of two objects occupying each location.

Procedure. The subjects were tested individually in the presence of the experimenter. The stimuli and instructions for the object location task were presented using a Macintosh II computer with a monochrome monitor. In the study phase, the subjects were instructed to place objects in various locations in different rooms of a house and to remember those locations for a later, unspecified test of memory. Eight practice trials were presented in random order, followed by 48 critical trials presented in random order. For each of these trials, the drawing of a household object was presented at the bottom of the monitor, and the drawing of the floor plan appeared above the object. The name of the room that contained the target location for that object was then highlighted. The subject was first asked to name aloud the household object and then to move the mouse to the highlighted room and click the mouse button. This action caused the floor plan to be erased and the drawing of the appropriate room to be displayed. The subject then clicked on the object, which was still present at the bottom of the display. With this click, a cue consisting of a large $X$ flashed twice in the target location and then remained on the monitor. The subject was then required to click on the cued location. The object was then erased from the bottom of the display and redrawn in the target location in place of the $X$. After a 1-sec viewing period, the room and object were erased, and the next trial began.

In the test phase, the 12 practice items were presented in random order ( 8 from the study phase and 4 nonstudied), followed by the 72 critical items, which were also presented in random order. The sub- 

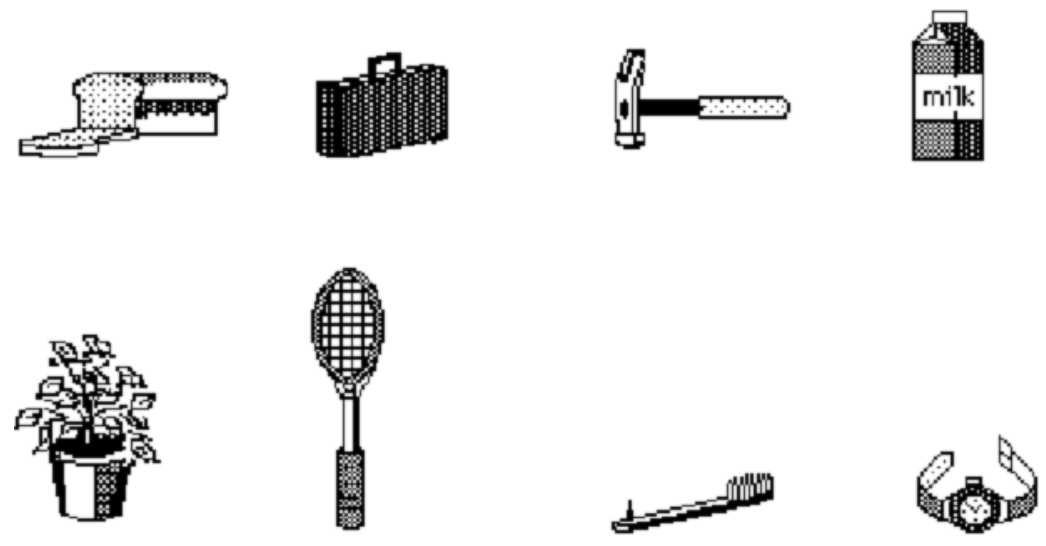

Figure 1. Examples of drawings of household objects.

jects were asked to search for the objects under inclusion and exclusion instructions. For those items assigned to the inclusion instruction condition, the subjects were told that each object occupied the same location as that in which they had placed it in the study phase, so they should try to recollect the old location and then search for the object in that location. If they could not remember the old location, they were told to search for the object in the first plausible location that came to mind. For items assigned to the exclusion condition, the subjects were told that the object was in a different location from that in which they had placed it in the study phase. For this task, they were instructed to recollect the old location and then search for the object in another plausible location. Again, they were told that if they could not remember the location in which they had placed it in the study phase, they should search for it in the first plausible location that came to mind. The subjects were informed that some of the objects were new, in that they had not been seen before in the experiment. For these objects, the subjects were told to choose the first plausible location that came to mind. These instructions were designed to encourage the subjects to directly retrieve old locations and thus were consistent with the boundary conditions for the assumption of independent influences of $C$ and $U$ (Jacoby, 1998).

The trials in the test phase were very similar to those in the study phase. The object was presented at the bottom of the monitor, with the floor plan appearing above it. The name of the appropriate room was highlighted on the floor plan. The subject clicked on the highlighted room, which erased the floor plan and displayed the drawing of the selected room, with the object still in view at the bottom of the display. The word SAME or DIFFERENT was presented beside the drawing of the object. This cue indicated whether the trial was an inclusion or an exclusion trial, respectively. No indication of the

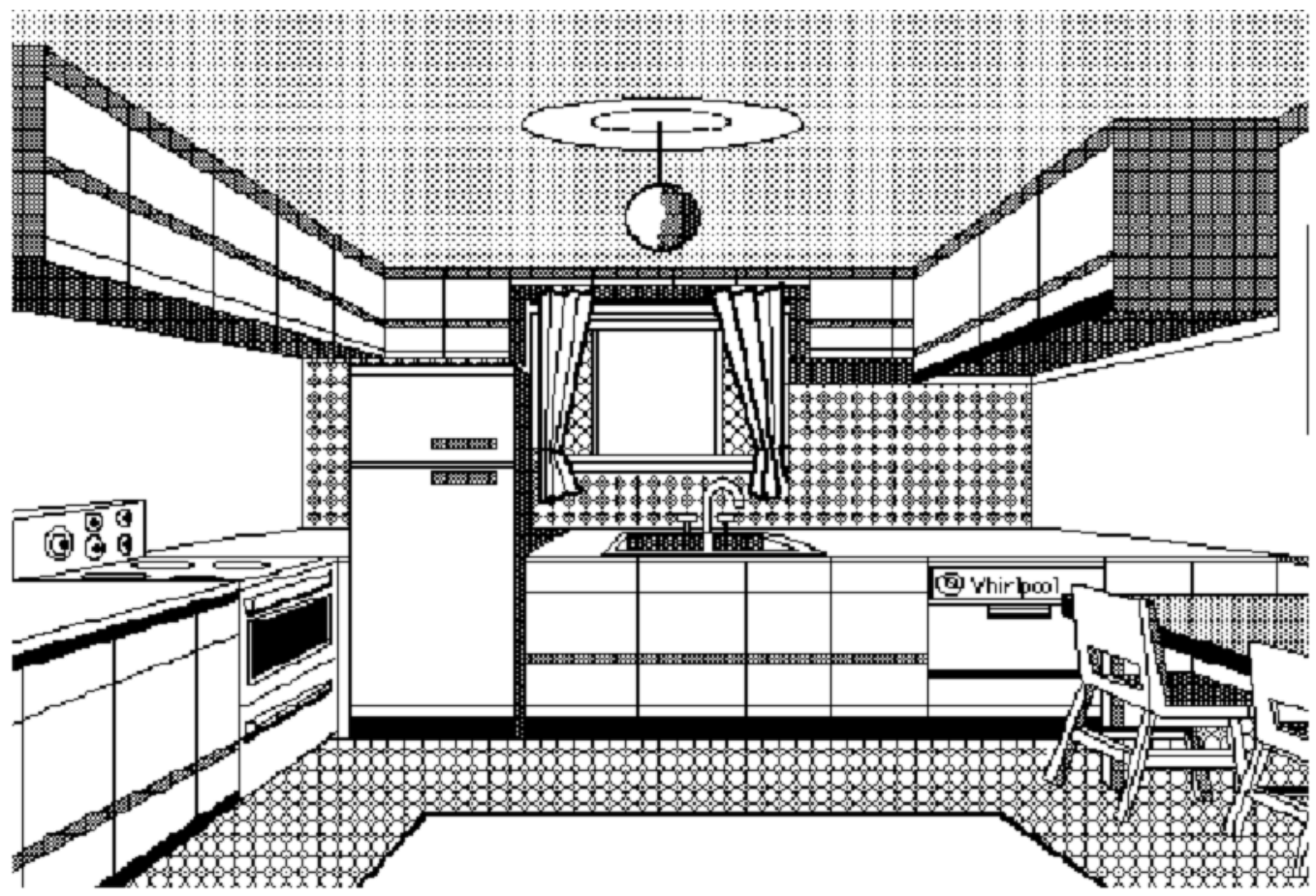

Figure 2. Drawing of one of the background rooms (kitchen). 


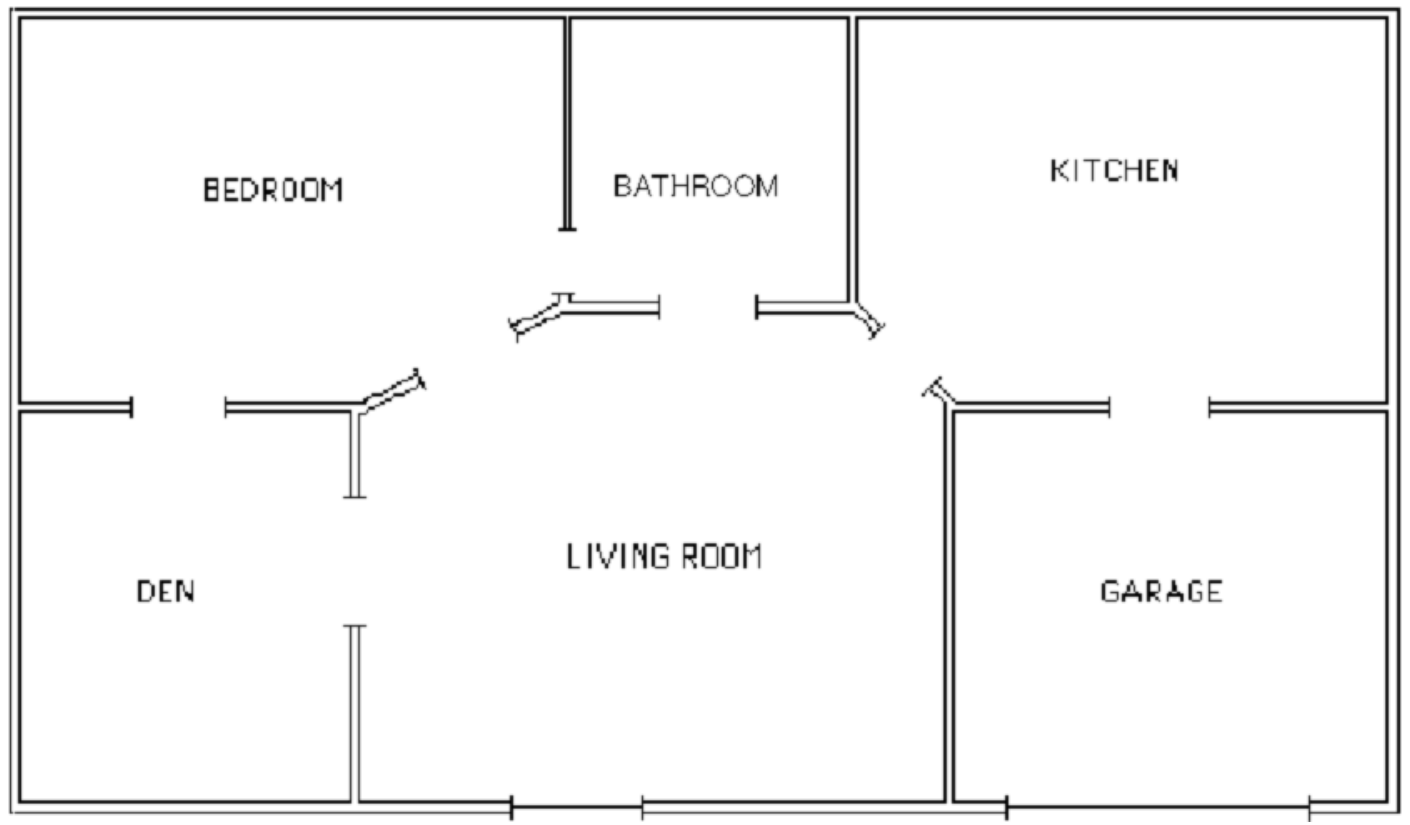

Figure 3. Drawing of the floor plan.

target location was visible in the display. The subject moved the mouse cursor around the drawing of the room, which caused the various locations within the room to become highlighted. The subject selected a location by clicking on that location when it was highlighted. For each object, only three predesignated locations accepted the click of the mouse, one of which was the old location. The two additional locations were high-probability choices for nonstudied objects in a pilot experiment that involved a similar search task. If the subjects clicked on a location that was not one of the three predesignated locations, the response was not accepted by the computer, and they were told by the experimenter to choose a different location. No feedback was provided during the test phase.

\section{Results and Discussion}

The dependent measure was the probability of selecting the target location. None of the subjects was successful at excluding the target location on every trial under exclusion instructions. This outcome is important because Jacoby, Toth, and Yonelinas (1993) argued that perfect exclusion scores can lead to an underestimation of $U$. Because no subject obtained a score of zero, this issue was not a concern in the present experiment.

The mean proportions of target locations selected under inclusion and exclusion instructions are presented in Table 1. For items in the nonstudied condition, the proportion of selected target locations serves as a baseline estimate of the likelihood of selecting that location in the absence of experience with that object in the present experiment. An analysis of variance (ANOVA) of the data for nonstudied objects, with instruction condition (inclusion and exclusion) as a within-subjects factor and age group (younger and older) as a between-subjects factor, was computed. The Type I error rate for this and all other ANOVAs reported here was set at .05. This ANOVA found no significant effects $\left(F_{\mathrm{S}}<1\right)$. The lack of effects in this analysis suggests that there was no systematic difference between the two age groups with respect to prototypical locations of the objects used here, nor was there a systematic influence of inclusion-exclusion instructions on selection of prototypical locations. Moreover, the lack of an effect of instructions on baseline items can be taken as an indication that the subjects were not using a generaterecognize strategy (Jacoby, 1998). If a generate-recognize strategy were present, performance on baseline items in the exclusion condition would be expected to be lower than that observed in the inclusion condition, because subjects would occasionally exclude new items on the basis of false recognition.

Separate ANOVAs demonstrated that for studied objects tested under inclusion instructions, younger subjects chose the studied location more often than did older subjects $\left[F(1,58)=18.24, M S_{\mathrm{e}}=0.019\right]$, whereas the reverse was true under exclusion instructions $[F(1,58)=18.20$, $\left.M S_{\mathrm{e}}=0.010\right]$. These differences indicate that the younger subjects were better able than the older subjects to use conscious recollection to guide selective search for the objects.

Estimates of $C$ and $U$ were computed for each subject, using the equations described above, in which it is assumed that these two influences operated independently. The mean estimates of $C$ and $U$ are shown in Table 1 . Separate ANOVAs for those estimates revealed that the younger subjects obtained a higher mean $C$ estimate than did the older subjects $\left[F(1,58)=26.12, M S_{\mathrm{e}}=0.039\right]$ but that the two groups did not reliably differ in their mean $U$ estimates $(F<1)$.

To determine whether the $U$ estimate obtained in the two age groups reflected a true influence of memory for 
Table 1

Mean Proportion of Target Locations Chosen and Estimates of Conscious and Unconscious Influences in Experiment 1

\begin{tabular}{|c|c|c|c|c|c|c|c|c|}
\hline \multirow[b]{3}{*}{ Condition and Estimates } & \multicolumn{4}{|c|}{ Younger Adults } & \multicolumn{4}{|c|}{ Older Adults } \\
\hline & \multicolumn{2}{|c|}{ Studied } & \multicolumn{2}{|c|}{$\overline{\text { Nonstudied }}$} & \multicolumn{2}{|c|}{ Studied } & \multicolumn{2}{|c|}{$\overline{\text { Nonstudied }}$} \\
\hline & $M$ & $S E$ & $M$ & $S E$ & $M$ & $\overline{S E}$ & $M$ & $\overline{S E}$ \\
\hline \multicolumn{9}{|l|}{ Instruction } \\
\hline Inclusion & .63 & .03 & .27 & .02 & .48 & .02 & .26 & .02 \\
\hline Exclusion & .18 & .01 & .26 & .02 & .29 & .02 & .28 & .02 \\
\hline \multicolumn{9}{|l|}{ Estimates from equations } \\
\hline Conscious $(C)$ & .45 & .03 & & & .19 & .04 & & \\
\hline Unconscious $(U)$ & .33 & .02 & & & .34 & .02 & & \\
\hline \multicolumn{9}{|c|}{ Estimates from multinomial model } \\
\hline Conscious $(C)$ & .45 & & & & .19 & & & \\
\hline Unconscious $(U)^{*}$ & .10 & & & & .10 & & & \\
\hline Guessing $(G)$ & .26 & & & & .28 & & & \\
\hline
\end{tabular}

the study episodes, rather than just preexperimental location preferences, an ANOVA was computed that compared the $U$ estimate with performance on nonstudied items (averaged across instructional condition), including age group as a factor. The only reliable effect was the main effect in which $U$ estimates were higher than the nonstudied baseline $\left[F(1,58)=13.03, M S_{\mathrm{e}}=0.010\right.$; other $\left.F \mathrm{~s}<1\right]$. The difference between the $U$ estimate and the nonstudied baseline indicates that there was a significant unconscious influence of memory for the specific study experience in which the subjects placed objects in designated locations.

These results provide support for the assumption that conscious and unconscious influences operate independently. Specifically, although conscious influences of memory for object location showed age-related differences, unconscious influences did not. This dissociation is consistent with previous research that has used the process-dissociation procedure to compare younger and older adults with respect to conscious and unconscious influences of memory for verbal material (e.g., Jacoby et al., 1997; Jennings \& Jacoby, 1993). The dissociation provides a source of validation of the estimates of conscious and unconscious influences of memory obtained from the independence equations. Moreover, the finding that the estimate of $U$ was reliably greater than the probability of placing nonstudied objects in target locations provides evidence for the claim that episodic experience can support an unconscious influence of memory for object location.

As an additional test of the independence assumption, a direct-retrieval (independence) version of the multinomial model developed by Buchner et al. (1995) and Jacoby (1998) was fit to the data. In this model (see the Appendix for details), it is assumed that there are three processes that can lead to selection of the target location: conscious recollection, unconscious influences of memory, and guessing. Each of these processes is assumed to operate independently. Unlike the estimates of $U$ produced above, the multinomial model separates out the contribution of guessing from those estimates, resulting in systematically smaller estimates.

In fitting the multinomial model to the observed data, we adopted two constraints on the parameters. First, $U$ was constrained to be equal for both age groups. Second, the estimate of guessing, $G$, was constrained to be equal in the inclusion and exclusion conditions (although it was allowed to vary across age groups). Model fitting was carried out using the Macintosh version of Hu's (1995) multinomial binary tree program, AppleTree (Rothkegel, 1997). The program provides maximum likelihood estimators for parameters, using an expectation-maximization algorithm (Hu \& Batchelder, 1994). Goodness of fit was measured using $G^{2}$, which is distributed approximately as $\chi^{2}$ (Read \& Cressie, 1988). A Type I error rate of .005 was adopted for testing the fit, to avoid rejecting the model because of only slight deviations from the observed results (Erdfelder \& Bredenkamp, 1998; Jacoby, 1998, 1999). Power to detect a small deviation from the data ( $w=.1$; Cohen, 1988) was greater than 99 .

The multinomial model provided a very good fit to the observed data $\left[G^{2}(3)=1.09, p>.75\right]$. The parameter estimates produced by this fit are shown in Table 1. As was expected, the estimate of $C$ was substantially larger for younger than for older adults. The estimate of $U$ (assumed to be equal for both age groups), with the influence of guessing removed, was somewhat smaller than the estimate provided by solving the independence equations, which includes guessing. The $95 \%$ confidence interval for the model's estimate (.06-.13), however, clearly indicated that the estimate for $U$ was greater than zero.

\section{EXPERIMENT 2}

In Experiment 2, we sought to demonstrate a dissociation between conscious and unconscious influences of memory for object location opposite to that found in Experiment 1 -one in which a manipulation affects the unconsciouscomponent while leaving the conscious com- 
ponent invariant. Taken together with the results of Experiment 1 , such a finding would establish a double dissociation between conscious and unconscious influences of memory and thus provide strong support for the assumption that the two processes operate independently.

We used a paradigm developed by Hay and Jacoby $(1996,1999)$ to investigate the effect of habit strength. By creating differential habit strength in responding to items in a training phase, Hay and Jacoby showed that the automatic influence of habit could have a variable influence on memory for subsequent episodes, even though conscious recollection of those episodes was invariant. In our adaptation of their procedure, we used a training phase to create habits of varying strength for putting objects in particular locations. Each object was placed multiple times in two different, plausible locations within a room. For objects assigned to the $75 \%$ condition, one location was used on $75 \%$ of the training trials, and the other location was used on $25 \%$ of the trials. This mixture was expected to create a relatively strong habit of placing the object in the typical $(75 \%)$ location. Objects in the $50 \%$ condition were placed equally often in the two designated locations, creating a rather weak habit.

The influence of these learned habits on memory for subsequent episodes was then assessed by presenting the subjects a set of object placement episodes and testing their memory for those episodes. In each of these studytest cycles, the subjects began by placing each member of a set of objects once in a designated location that corresponded to either the typical or the atypical location for that object. For objects in the 50\% condition, the classification of one location as typical and the other as atypical was arbitrary. The subjects were then tested by having them indicate the location in which each of the objects had been placed during this recent set of placement episodes. On each trial, only the two locations experienced during the training phase were available as response options.

Memory for the recent placement episodes should be influenced by two, presumably independent sources: conscious recollection of the location and the unconscious influence of the habit developed during training (which took place prior to the recent study episode). By assuming independence, a set of equations like those used in Experiment 1 can be applied to obtain quantitative estimates of conscious recollection $(R)$ and habit $(H)$. When an object is placed in its typical location in the study phase, the probability of selecting that location at test can be expressed as $T=R+H(1-R)$, where $T$ is the probability of selecting the typical location when that was the location used at study, $R$ is the probability of conscious recollection of the study location, and $H$ is the probability that habit formed during training will select the typical location. In this case, both $R$ and $H$ lead to the correct response because the studied location was the typical location. When an object is placed in its atypical location at study, however, the probability of selecting the typical location at test (which would be an incorrect response) can be expressed as $A=H(1-R)$, where $A$ is the probability of selecting the typical location even though the atypical location actually was used at study and $R$ and $H$ are the same as before. That is, only habit would direct the subject to the typical location-conscious recollection would correctly direct the subject to the atypical (studied) location. Estimation of $R$ and $H$ can be obtained by algebra as with the equations used in Experiment 1.

Following Hay and Jacoby (1996, 1999), in the test component of each study-test cycle, two additional items that had not been studied in that cycle were presented. Selection of the typical or atypical location for each of these nonstudied items should reflect only habit, because there was no study episode in that cycle to support conscious recollection. Performance on these items, then, was expected to provide converging evidence for the estimates of habit computed from the independence equations.

Hay and Jacoby $(1996,1999)$ found that the estimates of $H$ closely corresponded to the probabilities used to form the habit during training--a form of probability matching (e.g., Voss, Thompson, \& Keegan, 1959). They also found that the estimate of recollection was invariant across different levels of habit strength. We anticipated a similar outcome in Experiment 2. In particular, the estimate of $H$ was expected to approximate the probability with which typical locations were used during training (.75 in the $75 \%$ condition and .50 in the $50 \%$ condition), in keeping with probability matching. In addition, the estimate of $R$ was expected to be invariant across the two habit strength conditions.

\section{Method}

Subjects. Thirty subjects participated in Experiment 2. These subjects were recruited from the same source as the young subjects in Experiment 1. The data from 2 subjects were not included in the analyses because of ceiling performance on the study-test cycles. The ages of the remaining 28 subjects ranged from 18 to 24 years, with a median age of 19 years.

Design. This experiment consisted of a $2 \times 2$ factorial design, with training condition (75\% and 50\%) and study location (typical and atypical) as within-subjects factors.

Materials. The stimuli used in this experiment consisted of the six background rooms and a subset of 18 objects from the original set of 72 objects used in Experiment 1 . The 18 critical objects were chosen from the original set such that there were 3 objects per room. The objects were divided into two blocks of 9 objects so that no more than 2 objects from the same room were included in the same block. For each subject, one block of objects was assigned to the $75 \%$ training condition, and the other to the $50 \%$ condition. The assignment of the two blocks of objects to training conditions was counterbalanced across subjects. Two additional items were used as practice items at the beginning of the training phase.

For each critical object, two locations were chosen, and for each subject, one of these target locations was randomly chosen to be the typical location, and the other was the atypical location. The two locations chosen for each object were not shared with any other object in the experiment.

Procedure. The experiment was conducted using the same equipment as in Experiment 1 and consisted of two phases. The first was a training phase, in which the 18 critical objects were presented multiple times. On each trial, the subject was asked to place an object in one of its two locations. For the objects assigned to the $75 \%$ condition, the subject was asked to place the object in one location 
on $75 \%$ of the trials (the typical location) and in the other location on $25 \%$ of the trials (the atypical location). For those objects in the $50 \%$ condition, the subjects placed the object in the typical location on $50 \%$ of the trials and in the atypical location on $50 \%$ of the trials. For this condition, the typical-atypical distinction was arbitrary.

The 2 practice objects were presented first; then the critical objects were presented in five blocks of 72 trials. In each block, each of the 18 objects was presented four times in a random order for a total of 20 presentations per object across the five blocks. The frequency of using an object's typical and atypical locations within a block matched the object's training condition (3:1 for the $75 \%$ condition and 2:2 for the $50 \%$ condition). Training trials were very similar to the trials in the study phase of Experiment 1 . The only exception was that the drawing of the floor plan was presented only once, just prior to the first training trial, to acquaint the subjects with the various rooms being used. The floor plan was not presented again during the experiment. At the start of each trial, the drawing of the object and the room appeared on the subject's monitor. The subject named the object aloud, then clicked on the object, using the computer's mouse cursor. This action caused the cue $(X)$ to appear in the target location. The subject then clicked on the cued location, which caused the object to be erased and redrawn in the target location. The subjects were instructed that they would see each of the objects numerous times and that they were to continue placing them in the cued locations. They were further told that the purpose of the training phase was to provide them with practice in placing the objects in the designated locations.

The second phase of the experiment consisted of a series of 18 study-test cycles. The study component of each cycle consisted of the presentation of eight critical objects, four from each of the two training conditions. For the objects in the $75 \%$ condition, three appeared in their typical locations, and one appeared in its atypical location. For objects in the $50 \%$ condition, two each appeared in their typical and atypical locations. Across the 18 study-test cycles, each of the 18 critical items appeared for study eight times. The relative frequency of appearance of an object in its typical and atypical locations during these study episodes matched the relative training frequencies. On each trial, the drawing of the room was presented on the monitor, and the target location was cued by the presentation of an $X$ for $1 \mathrm{sec}$. The cue was then erased, and the drawing of the object appeared in that location. The object remained on the monitor for $1 \mathrm{sec}$ and then was erased. The next trial then began automatically. The subjects were instructed to remember where each object had appeared for a later memory test.

After each study list, there was a short distractor task in which a number between 30 and 100 appeared on the screen for $1 \mathrm{sec}$ and then was erased. The subject counted backward by threes from that number for $8 \mathrm{sec}$; then the test trials began. Ten test trials were presented in random order in each study-test cycle. Eight of these trials involved objects from that cycle's study list, and two involved objects that had not been presented in that study list. One of the nonstudied items was from the $75 \%$ condition, and one was from the $50 \%$ condition. Across the 18 study-test cycles, each of the 18 critical objects appeared twice as a nonstudied item. The subjects were asked to choose the location in which the objects had appeared in the study list. They were further instructed that if they could not remember the location in which the object had appeared, they should choose the first location that came to mind. The subjects were also warned that some objects presented in the test did not appear in the study list and that, for these objects, they should also choose the first location that came to mind. These test trials proceeded in the same manner as they did in the test phase of Experiment 1, except that only two locations became highlighted and accepted a click of the mouse: the typical and atypical locations for the object being tested. Thus, the subjects were forced to choose between these two locations. No feedback was provided regarding the correctness of these choices.

\section{Results and Discussion}

The mean proportion of trials on which the subjects selected an object's typical location on test trials are shown in Table 2 as a function of training condition and whether the object's studied location was the typical or the atypical one. To determine whether training condition had a significant influence on memory for subsequent object placement episodes, an ANOVA was conducted on test performance data (probability of selecting the typical location), with training condition ( $75 \%$ and $50 \%)$ and studied location (typical and atypical) as within-subjects factors. The ANOVA revealed that the $75 \%$ training condition led to a higher proportion of typical responses at test $\left[F(1,27)=29.01, M S_{\mathrm{e}}=0.005\right]$, and that typical responses were more likely when the object had actually been placed in its typical location during the study phase $\left[F(1,27)=783.75, M S_{\mathrm{e}}=0.018\right]$. The interaction between these two factors was not significant $(F<1)$.

A higher proportion of typical responses was found in the $75 \%$ training condition, regardless of where an object was actually studied. This finding reflects two important influences of habit. First, when an object is placed in its typical location on a particular episode, habit supports correct performance in locating that object. Second, finding an object that has been placed in an atypical location can be hampered by a strong habit that associates that object with a different location. This latter influence of habit is a form of memory slip and is particularly likely to occur when strong habits are involved (Hay \& Jacoby, 1996).

Assuming independence between conscious recollection of object location and unconscious influences of habit, we applied the equations described above to obtain quantitative estimates of recollection and habit. The mean estimates of these two parameters are shown in Table 2. Separate ANOVAs were conducted on these estimates to determine how recollection and habit varied as a function of training condition ( $75 \%$ vs. $50 \%)$. The estimate of recollection did not significantly differ across the two training conditions $(F<1)$, but the estimate of habit was reliably greater in the $75 \%$ condition than in the $50 \%$ condition $\left[F(1,27)=60.14, M S_{\mathrm{e}}=0.018\right]$. Moreover, the estimates of habit obtained in the two training conditions closely approximated the proportions used in the training phase. This tendency toward probability matching replicates the pattern of habit estimates obtained by Hay and Jacoby $(1996,1999)$ for word-fragment completion. Importantly, the finding that training condition did not affect estimates of recollection but did affect estimates of habit constitutes a dissociation between conscious and unconscious influences of memory for object location. Taken together with the dissociation found in Experiment 1 , this result establishes a double dissociation between these two influences and provides strong support for the assumption that they operate independently.

In contrast to the Hay and Jacoby $(1996,1999)$ results, the nonstudied items in Experiment 2 did not produce proportions of typical responses that differed significantly as a function of training condition $(F<1.2)$. Con- 
Table 2

Mean Proportion of Typical Locations Chosen on Test Trials and Estimates of Recollection and Habit in Experiment 2

\begin{tabular}{|c|c|c|c|c|}
\hline \multirow[b]{3}{*}{ Condition and Estimates } & \multicolumn{4}{|c|}{ Training Condition } \\
\hline & \multicolumn{2}{|c|}{$75 \%$} & \multicolumn{2}{|c|}{$50 \%$} \\
\hline & $M$ & $S E$ & $M$ & $S E$ \\
\hline \multicolumn{5}{|l|}{ Studied location } \\
\hline Typical & .94 & .01 & .86 & .01 \\
\hline Atypical & .21 & .03 & .15 & .01 \\
\hline Nonstudied & .54 & .03 & .51 & .03 \\
\hline \multicolumn{5}{|l|}{ Estimates from equations } \\
\hline Recollection $(R)$ & .73 & .03 & .71 & .02 \\
\hline Habit $(H)$ & .78 & .03 & .49 & .03 \\
\hline \multicolumn{5}{|c|}{ Estimates from multinomial model } \\
\hline Recollection $(R)^{*}$ & .72 & & .72 & \\
\hline Habit $(H)$ & .50 & & .00 & \\
\hline Guessing $(G)$ & .54 & & .51 & \\
\hline
\end{tabular}

*Estimate for $R$ was constrained in the multinomial model to be equal for both training conditions.

sequently, these proportions also did not approximate the habit estimates for the $75 \%$ and $50 \%$ training conditions obtained using the independence equations. The purpose of testing nonstudied items was to provide converging evidence for those estimates of habit. Nonstudied items were not presented in the study lists, so performance on those items could not be affected by conscious recollection, but only by habit created during training or by guessing. We suspect that in the Hay and Jacoby experiments, in which test stimuli consisted of word fragments, subjects were likely to have had difficulty remembering whether an item had been presented in the study list and would therefore have applied similar remembering processes to both studied and nonstudied items. This process would have given the experimentally trained habit a full opportunity to exert its influence on fragment completion. In Experiment 2, however, the stimuli were easily identifiable objects, so the subjects may have readily noticed whether an object had appeared in the study list. For those objects identified as nonstudied, the subjects may not have relied on memory for recent study episodes when choosing a location for those objects in the test and, instead, might simply have made a guess or used other information, such as where they keep the object in their own home. Under these circumstances, the habits formed during training would be unlikely to influence performance on the nonstudied objects.

As a further test of the hypothesis that recollection and habit operate independently in this paradigm, a multinomial model consisting of three independent processesconscious recollection $(R)$, habit $(H)$, and guessing $(G)$ was constructed. Full specification of this model is provided in the Appendix. The model was fit to the data of Experiment 2, using the same procedures and criteria as those described in Experiment 1, with the constraint that $R$ be equal in both habit strength conditions. The resulting fit was very good $\left[G^{2}(1)=0.60, p>.40\right]$, providing further evidence for the assumption that conscious and unconscious influences of memory for location can operate independently. The estimates of the three parameters produced by this fit are shown in Table 2. As in Experiment 1, separating the estimate of guessing from the automatic influence of memory (in this case, $H$ ) resulted in systematically lower estimates of that influence than was found with the independence equations. The zero estimate for $H$ in the $50 \%$ condition reflects the fact that habit in this case is entirely determined by an equiprobable choice between alternatives. To the extent that the choice between the alternatives differentially favors one over the other, the model's estimate of $H$ will rise above zero, as was seen in the $75 \%$ condition. An alternative model that did not include a separate guessing parameter produced a similarly good fit to the data and estimated $H$ at .77 and .51 for the $75 \%$ and $50 \%$ training conditions, respectively.

\section{GENERAL DISCUSSION}

The experiments reported here were designed to provide evidence for two different influences of memoryone conscious and the other unconscious--for object location. On the initial simplifying assumption that these two influences operate independently, we used Jacoby's (1991) process-dissociation procedure to establish a double dissociation between these two influences. In Experiment 1 , estimates of conscious recollection of object location were lower for older than for younger adults, whereas unconscious influences showed no age-related differences. In Experiment 2, we found the opposite dissociation, in which unconscious, but not conscious, influences of memory were affected by habit strength. These findings have important implications for the Hasher and Zacks (1979) proposal regarding automatic encoding of spatial information and for the assumption that conscious and unconscious influences of memory operate independently.

Hasher and Zacks (1979) argued that because spatial information is encoded automatically, it should not be affected by developmental trends. A consistent finding, however, is that older adults do perform worse than younger adults on tasks that assess memory for spatial location (e.g., Light \& Zelinski, 1983; Naveh-Benjamin, 1987, 1988). This evidence, however, has been obtained using direct tests that arguably are most strongly determined by conscious influences of memory. The demonstration in Experiment 1 that unconscious influences of memory for location were comparable across young and older adults, whereas age-related deficits were observed for conscious recollection, highlights the importance of distinguishing these two aspects of memory when assessing age-related differences. In the case of memory for location or spatial information, it appears that unconscious influences operate with similar success in older and younger adults, in keeping with the Hasher and Zacks proposal. Contrary to that proposal, however, processes involved in conscious recollection, including strategic encoding processes, are likely to lead to age-related differences in favor of younger adults (e.g., Naveh-Benjamin, 1987). 
An important benefit of the process-dissociation procedure lies in the opportunity to obtain quantitative estimates of conscious and unconscious influences of memory. In the process-dissociation procedure, the estimation process depends on the assumption that conscious and unconscious influences of memory operate independently (Jacoby, 1991). We took three steps to ensure that the independence assumption was a reasonable one in analyzing the present experiments. First, we attempted to adhere to the boundary conditions described by Jacoby (1998) in presenting direct retrieval instructions to subjects and establishing in Experiment 1 that performance on nonstudied items was consistent across instructional conditions. Second, we established a double dissociation between estimates of conscious and unconscious influences that followed a pattern that was predictable from earlier research based on direct and indirect tests of memory. Finally, we successfully fit to the results of both experiments a multinomial model that assumed independent operation of conscious and unconscious influences of memory.

Although dissociations of the type demonstrated in the present set of experiments support the assumption of independence, they can also be explained by other assumptions regarding the relation between conscious and unconscious influences of memory. Bodner, Masson, and Caldwell (2000) have shown that for the word-stem completion task, dissociations between estimates of conscious and unconscious influences of memory can be accounted for by a generate-recognize model in which a redundancy relation holds between those two influences (e.g., Joordens \& Merikle, 1993). In that model, it is assumed that unconscious influences contribute to the generation of possible completions and conscious influences guide selection among those completions. Thus, consciously remembered completions are always a (redundant) subset of unconsciously generated completions. A similar relation may hold in the case of memory for object location, and future research should be directed toward that possibility. At stake is the issue of whether the relation between conscious and unconscious influences of memory is fundamentally the same across various cognitive tasks or whether that relation varies in a principled way. The present experiments, however, serve to establish initial evidence in support of an independence relation between conscious and unconscious influences of memory for object location.

\section{REFERENCES}

Anooshian, L. J., \& SeIBert,P. S. (1996). Diversity within spatial cognition: Memory processes underlying place recognition. Applied Cognitive Psychology, 10, 281-299.

Bodner, G. E., Masson, M. E. J., \& Caldwell, J. I. (2000). Evidence for a generate-recognize model of episodic influences on word-stem completion. Journal of Experimental Psychology: Learning, Memory, \& Cognition, 26, 267-293.

Brown, J. I., Bennett, J. M., \& Hanna, G. (1981). The Nelson-Denny Reading Test. Chicago: Riverside.

Buchner, A., Erdfelder, E., \& VAterrodt-Plünnecke, B. (1995). Toward unbiased measurement of conscious and unconscious memory processes within the process dissociation framework. Journal of Experimental Psychology: General, 124, 137-160.
Chiarello, C., \& Hoyer, W. J. (1988). Adult age differences in implicit and explicit memory: Time course and encoding effects. Psychology \& Aging, 3, 358-366.

CoHEn, J. (1988). Statistical power analysis for the behavioral sciences (2nd ed.). Hillsdale, NJ: Erlbaum.

Curran, T., \& Hintzman, D. L. (1995). Violations of the independence assumption in process dissociation. Journal of Experimental Psychology: Learning, Memory, \& Cognition, 21, 531-547.

Curran, T., \& Hintzman, D. L. (1997). Consequences and causes of correlations in process dissociations. Journal of Experimental Psychology: Learning, Memory, \& Cognition, 23, 496-504.

ERDFELDER, E., \& BREDENKAMP, J. (1998). Recognition of script-typical versus script-atypical information: Effects of cognitive elaboration. Memory \& Cognition, 26, 922-938.

Graf, P., \& Komatsu, S. (1994). Process dissociation procedure: Handle with caution! European Journal of Cognitive Psychology, 6, 113129.

Greene, R. L. (1986). Word stems as cues in recall and completion tasks. Quarterly Journal of Experimental Psychology, 38A, 663-673.

HASHER, L., \& ZACKs, T. T. (1979). Automatic and effortful processes in memory. Journal of Experimental Psychology: General, 108, 356388

HAY, J. F., \& JACOBY, L. L. (1996). Separating habit and recollection: Memory slips, process dissociations, and probability matching. Journal of Experimental Psychology: Learning, Memory, \& Cognition, 22, 1323-1335.

HAY, J. F., \& JACOBY, L. L. (1999). Separating habit and recollection in young and older adults: Effects of elaborative processing and distinctiveness. Psychology \& Aging, 14, 122-134.

Hu, X. (1995). Statistical inference program for multinomial binary tree models [Computer software]. Irvine, CA: University of California.

Hu, X., \& Batchelder, W. H. (1994). The statistical analysis of general processing tree models with the EM algorithm. Psychometrika, 59, 21-48.

Hultsch, D. F., Masson, M. E. J., \& Small, B. J. (1991). Adult age differences in direct and indirect tests of memory. Journals of Gerontology: Psychological Sciences, 46, P22-P30.

JACOBY, L. L. (1991). A process dissociation framework: Separating automatic from intentional uses of memory. Journal of Memory \& Language, 30, 513-541.

JACOBY, L. L. (1998). Invariance in automatic influences of memory: Toward a user's guide for the process-dissociation procedure. Journal of Experimental Psychology: Learning, Memory, \& Cognition, 24, 3-26.

JACOBY, L. L. (1999). Ironic effects of repetition: Measuring age-related differences in memory. Journal of Experimental Psychology: Learning, Memory, \& Cognition, 25, 3-22.

JACOBY, L. L., \& DAllas, M. (1981). On the relationship between autobiographical memory and perceptual learning. Journal of Experimental Psychology: General, 110, 306-340.

JACoBy, L. L., Jennings, J. M., \& Hay, J. F. (1996). Dissociating automatic and consciously-controlled processes: Implications for diagnosis and rehabilitation of memory deficits. In D. J. Herrmann, C. L. McEvoy, C. Hertzog, P. Hertel, \& M. K. Johnson (Eds.), Basic and applied memory research: Theory in context (Vol. 1, pp. 161-193). Hillsdale, NJ: Erlbaum.

JACOBY, L. L., LindSAY, D. S., \& Toth, J. P. (1992). Unconscious influences revealed: Attention, awareness, and control. American Psychologist, 47, 802-809.

JACoby, L. L., Tотн, J. P., \& Yonelinas, A. P. (1993). Separating conscious and unconscious influences of memory: Measuring recollection. Journal of Experimental Psychology: General, 122, 139-154.

Jacoby, L. L., Yonelinas, A. P., \& Jennings, J. M. (1997). The relation between conscious and unconscious (automatic) influences: A declaration of independence. In J. D. Cohen \& J. W. Schooler (Eds.), Scientific approaches to consciousness (pp. 13-47). Mahwah, NJ: Erlbaum.

JenNings, J. M., \& JACOBY, L. L. (1993). Automatic versus intentional uses of memory: Aging, attention, and control. Psychology \& Aging, 8, 283-293.

JENNINGS, J. M., \& JACOBY, L. L. (1997). An opposition procedure for 
detecting age-related deficits in recollection: Telling effects of repetition. Psychology \& Aging, 12, 352-361.

Joordens, S., \& MerikLe, P. M. (1993). Independence or redundancy? Two models of conscious and unconscious influences. Journal of Experimental Psychology: General, 122, 462-467.

Light, L. L., \& Singh, A. (1987). Implicit and explicit memory in young and older adults. Journal of Experimental Psychology: Learning, Memory, \& Cognition, 13, 531-541.

Light, L. L., \& ZeLINSKI, E. M. (1983). Memory for spatial information in young and old adults. Developmental Psychology, 19, 901-906.

Mandler, J. M., Seegmiller, D., \& Day, J. (1977). On the coding of spatial information. Memory \& Cognition, 5, 10-16.

NaVeh-Benjamin, M. (1987). Coding of spatial location information: An automatic process? Journal of Experimental Psychology: Learning, Memory, \& Cognition, 13, 595-605.

Naveh-Benjamin, M. (1988). Recognition memory of spatial location information: Another failure to support automaticity. Memory \& Cognition, 16, 437-445.

PARK, D. C., \& MAson, D. A. (1982). Is there evidence for automatic processing of spatial and color attributes present in pictures and words? Memory \& Cognition, 10, 76-81.

PARKin, A. J., ReID, T. K., \& Russo, R. (1990). On the differential nature of implicit and explicit memory. Memory \& Cognition, 18, 507 514.

READ, T. R. C., \& CRessie, N. A. C. (1988). Goodness-of-fit statistics for discrete multivariate data. New York: Springer-Verlag.

RoTHKEgEL, R. (1997). AppleTree [Computer software]. Trier: University of Trier.

Shadoin, A. L., \& Ellis, N. R. (1992). Automatic processing of memory for spatial location. Bulletin of the Psychonomic Society, 30, 55 57.

Small, B. J., Hultsch, D. F., \& Masson, M. E. J. (1995). Adult age differences in perceptually based, but not conceptually based implicit tests of memory. Journals of Gerontology: Psychological Sciences \& Social Sciences, 50B, P162-P170.

Voss, J. F., Thompson, C. P., \& KeEgan, J. H. (1959). Acquisition of probabilistic paired associates as a function of $S-R_{1}, S-R_{2}$ probability. Journal of Experimental Psychology, 58, 390-399.

\section{APPENDIX \\ Description of Direct-Retrieval Multinomial Model}

The multinomial model used to fit the data obtained in the experiments reported here was the direct-retrieval version of the multinomial model used by Jacoby (1998). The specifications of the model, as applied to the data from Experiments 1 and 2, are shown in Table A1. In Experiment 1, the model had three parameters: $C$, the probability of conscious recollection of the target location; $U$, the probability that unconscious influences of memory produce the target location; and $G$, the probability that the target location is produced by a guessing process that is independent of prior study. In Experiment 2, the three parameters were the following: $R$, the probability that the studied location is consciously recollected; $H$, the probability that the typical location is produced by the unconscious influence of habit; and $G$, the probability that the typical location is produced by a guessing process that is independent of the study episode.

Table A1

Direct-Retrieval Multinomial Model for Experiments 1 and 2

\begin{tabular}{|c|c|c|c|}
\hline \multicolumn{2}{|l|}{ Experiment 1} & \multicolumn{2}{|l|}{ Experiment 2} \\
\hline $\begin{array}{l}\text { Instruction Condition, } \\
\text { Item, and Parameters }\end{array}$ & Response & $\begin{array}{l}\text { Study Condition } \\
\text { and Parameters }\end{array}$ & Response \\
\hline $\begin{array}{l}\text { Inclusion } \\
\text { Studied } \\
\qquad \begin{array}{l}C U \\
C(1-U) \\
\quad(1-C) U \\
\quad(1-C)(1-U) G \\
\quad(1-C)(1-U)(1-G\end{array}\end{array}$ & $\begin{array}{l}\text { target } \\
\text { target } \\
\text { target } \\
\text { target } \\
\text { nontarget }\end{array}$ & $\begin{array}{l}\text { Study typical } \\
\qquad \begin{array}{l}R H \\
R(1-H) \\
\quad(1-R) H \\
\quad(1-R)(1-H) G \\
\quad(1-R)(1-H)(1-G)\end{array}\end{array}$ & $\begin{array}{l}\text { target } \\
\text { target } \\
\text { target } \\
\text { target } \\
\text { nontarget }\end{array}$ \\
\hline $\begin{array}{l}\text { New } \\
\qquad G \\
\quad(1-G)\end{array}$ & $\begin{array}{l}\text { target } \\
\text { nontarget }\end{array}$ & $\begin{array}{l}\text { Study atypical } \\
\qquad R H \\
\quad R(1-H)\end{array}$ & $\begin{array}{l}\text { nontarget } \\
\text { nontarget }\end{array}$ \\
\hline $\begin{array}{l}\text { Exclusion } \\
\text { Studied } \\
\qquad \begin{array}{l}C U \\
\quad C(1-U) \\
\quad(1-C) U \\
\quad(1-C)(1-U) G \\
\quad(1-C)(1-U)(1-G\end{array}\end{array}$ & $\begin{array}{l}\text { nontarget } \\
\text { nontarget } \\
\text { target } \\
\text { target } \\
\text { nontarget }\end{array}$ & $\begin{array}{l}\quad(1-R) H \\
\quad(1-R)(1-H) G \\
\quad(1-R)(1-H)(1-G) \\
\text { Nonstudied } \\
\quad G \\
\quad(1-G)\end{array}$ & $\begin{array}{l}\text { target } \\
\text { target } \\
\text { nontarget }\end{array}$ \\
\hline $\begin{array}{l}\text { New } \\
\qquad G \\
\quad(1-G)\end{array}$ & $\begin{array}{l}\text { target } \\
\text { nontarget }\end{array}$ & & \\
\hline
\end{tabular}

(Manuscript received June 2, 1999; revision accepted for publication June 21, 2000.) 